\title{
CORRIGENDUM
}

\section{Cytokines in affective disorders and schizophrenia: new clinical and genetic findings}

P Prolo and J Licinio. Mol Psychiatry 1999; 4: 109-111

The sentence 'In this regard it is noteworthy that A nisman et al found that IL-1 $\beta$ was more dysregulated in depression than in dysthymia, regardless of typical or atypical symptoms' is incorrect. The correct sentence is 'In this regard it is noteworthy that Anisman et al found that IL- $1 \beta$ was more dysregulated in dysthymia than in depression, regardless of typical or atypical symptoms'. 